\title{
Criação e validação de um fluxograma de encaminhamento do paciente idoso para
}

\section{uso do médico não-geriatra}

\author{
Creation and validation of an elderly patient referral flowchart for no geriatric practitioner use \\ Creación y validación de un diagrama de flujo de derivación de pacientes ancianos para uso de \\ médico no geriatra
}

Recebido: 06/09/2021 | Revisado: 10/09/2021 | Aceito: 14/09/2021 | Publicado: 15/09/2021

\author{
Tanise Nazaré Maia Costa \\ ORCID: https://orcid.org/0000-0003-1319-5591 \\ Centro Universitário do Estado do Pará, Brasil \\ E-mail: tanise.costa@prof.cesupa.br \\ Cristiane Ribeiro Maués \\ ORCID: https://orcid.org/ 0000-0002-2338-7944 \\ Centro Universitário do Estado do Pará, Brasil \\ E-mail: crismaues@gmail.com \\ Amanda dos Santos Duarte \\ ORCID: https://orcid.org/ 0000-0003-2214-2870 \\ Centro Universitário do Estado do Pará, Brasil \\ E-mail: amandadsduarte@ hotmail.com \\ Felipe Aguiar Parente \\ ORCID: https://orcid.org/ 0000-0002-3989-8722 \\ Centro Universitário do Estado do Pará, Brasil \\ E-mail: parente.fnmv@gmail.com
}

\begin{abstract}
Resumo
A população mundial vem sofrendo transformações no âmbito social, político e tecnológico, caracterizando a transição demográfica, o qual modificou a pirâmide etária, com envelhecimento em maior velocidade do que a natalidade. Essas mudanças geraram impactos no atendimento em saúde. Com isso, objetivou-se criar e validar um fluxograma de encaminhamento do paciente idoso para uso do médico não geriatra. Esta pesquisa foi constituída por três etapas. A primeira consistiu em um estudo transversal analítico, através de um questionário autoaplicável para 49 médicos não-geriatras, o qual avaliou o grau de conhecimento do profissional médico quanto ao encaminhamento correto; a segunda, na criação do Fluxograma para orientar os profissionais não-geriatras ao encaminhamento correto para o ambulatório de saúde do idoso; e, na terceira etapa, realizado questionário aos médicos especialistas para validar o fluxograma criado. Com análise de 44 questionários, $65,9 \%$ dos profissionais encaminharam um idoso em algum momento da carreira. Demonstrou-se que $43,2 \%$ dos participantes acertaram quatro questões, tendo maior grau de conhecimento quanto à síndrome de incapacidade cognitiva e menor de instabilidade postural. Foi desenvolvido um fluxograma de encaminhamento para orientação que foi validado pela opinião dos especialistas com aprovação de $84,4 \%$. Mediante resultados obtidos, a implementação do fluxograma padronizou e contribuiu para uma decisão clínica mais acurada, rápida e eficiente, a fim de tornar mais eficiente o atendimento das demandas do ambulatório de saúde do idoso e aumentar o número de vagas para atendimento de idosos que realmente necessitam do atendimento especializado.
\end{abstract}

Palavras-chave: Idoso; Assistência integral à saúde; Fluxograma.

\begin{abstract}
The world population has been undergoing changes in the social, political and technological spheres, characterizing the demographic transition, which changed the age pyramid, aging at a faster rate than the birth. These changes had an impact on health care. Thus, the objective was to create and validate a flowchart for the criterion of elderly patients for use by no geriatric doctor. This research included three stages. The first be composed of an analytical crosssectional study, using a self-administered questionnaire for 49 no geriatrics practitioners, which assessed the doctor's level of knowledge regarding the correct referral; the second, in the creation of the Flowchart to guide general practitioners to the correct referral to the elderly health clinic; and, in the third stage, a questionnaire was conducted with specialist physicians to validate the flowchart created. With the investigation of 44 questionnaires, $65.9 \%$ of professionals referred an elderly person at some point in their career. It was shown that $43.2 \%$ of the participants got 4 questions right, having more knowledge about the cognitive disability syndrome and less about postural instability. A referral flowchart for guidance was developed and validated by expert opinion with $84.4 \%$ approval. Established of
\end{abstract}


the results obtained, the implementation of the flowchart standardized and contributed to a more accurate, quick and efficient clinical decision, for the purpose of make more efficient the meeting of the requests of the elderly health clinic and increase the numeral of vacancies for the protection of the elderly who actually need specialized care.

Keywords: Aged; Comprehensive health care; Workflow.

\section{Resumen}

La población mundial ha venido experimentando cambios en los ámbitos social, político y tecnológico, caracterizando la transición demográfica, que cambió la pirámide de edad, envejeciendo a un ritmo más rápido que la natalidad. Estos cambios tuvieron un impacto en la atención médica. Así, el objetivo fue crear y validar un diagrama de flujo para la derivación de pacientes ancianos para uso del médico de cabecera. Esta investigación consta de tres etapas. El primero consistió en un estudio analítico transversal, mediante un cuestionario autoadministrado a 49 médicos no geriatras, que evaluó el nivel de conocimiento del médico sobre la correcta derivación; el segundo, en la creación del Diagrama de flujo para orientar a los médicos generales a la correcta derivación a la clínica de salud de la tercera edad; y, en la tercera etapa, se realizó un cuestionario con médicos especialistas para validar el diagrama de flujo elaborado. Con el análisis de 44 cuestionarios, el $65,9 \%$ de los profesionales remitieron a una persona mayor en algún momento de su carrera. Se demostró que el 43,2\% de los participantes acertaron 4 preguntas, teniendo más conocimiento sobre el síndrome de discapacidad cognitiva y menos sobre la inestabilidad postural. Se desarrolló un diagrama de flujo de remisión para orientación y se validó mediante la opinión de un experto con una aprobación del 84,4\%. Sobre la base de los resultados obtenidos, la implementación del diagrama de flujo estandarizó y contribuyó a una decisión clínica más certera, rápida y eficiente, con el fin de hacer más eficiente el cumplimiento de las demandas de la clínica de salud de la tercera edad y aumentar la cantidad de vacantes para la atención. de los ancianos que realmente necesitan cuidados especializados.

Palabras clave: Anciano; Atención integral de salud; Flujo de trabajo.

\section{Introdução}

A população mundial com o decorrer da história passou por mudanças no âmbito social, político e tecnológico, o que ocasionou um fenômeno global conhecido como transição demográfica, o qual transformou a pirâmide etária, aumentado o seu ápice e diminuindo sua base, isto é, a população mundial está envelhecendo em maior velocidade do que nascer (Freitas \& Py, 2017; World Health Organization, 2015; Sandri, Raupp, Ploner \& Nunes, 2019).

Dessa forma, o Brasil apesar de ser um país em desenvolvimento, também foi atingido por esse fenômeno, denotando que a expectativa de vida chegasse em 76,3 anos no ano de 2018, segundo o Instituto Brasileiro de Geografia e Estatística (IBGE, 2019).

Essa mudança sociodemográfica no país, gerou impactos importantes, o que inclui o atendimento à saúde, já que o padrão de patologias prevalentes que antes eram doenças infecciosas como o sarampo, tuberculose e outras, passou a ser patologias crônicas associadas a senilidade, como a hipertensão arterial, demências, diabetes mellitus e muitas outras doenças que têm sua incidência aumentada em idades superiores a 65 anos (Damasceno \& Chirell, 2019).

Outro ponto importante a ser esclarecido é a definição atual de idoso, o qual é conceituado pela Organização Mundial da Saúde (OMS), como toda pessoa com 65 ou mais anos de idade. No caso do Brasil, o Estatuto do Idoso preconiza à pessoa idosa como aquele indivíduo com 60 ou mais anos de idade (World Health Organization, 2015; Ministério da Saúde, 2013). Assim, para atender as necessidades da população idosa, o governo federal brasileiro sancionou a Lei federal número 8.842, de 04 de janeiro de 1994, que institui a Política Nacional do Idoso, que visa "assegurar os direitos sociais do idoso, criando condições para promover sua autonomia, integração e participação efetiva na sociedade" (Brasil, 1994).

Com esse envelhecimento populacional, encontrou-se maior importância nos termos autonomia e independência. A autonomia é a capacidade de cada indivíduo de tomar decisões e ter comando sobre suas próprias ações, estabelecendo e cumprindo suas próprias regras, sendo que esta é dependente da cognição e do humor desse indivíduo. Já independência significa a capacidade de realizar algo com os próprios meios, dependente da mobilidade e comunicação (Brasil, 2014; Santos et al, 2018).

Sendo assim cognição, humor, mobilidade e comunicação são os quatros domínios essenciais para garantir que um 
idoso seja capaz de funcionar sozinho, de forma independente e autônoma, mesmo ele possuindo alguma comorbidade (Brasil, 2014; Damasceno \& Chirell, 2019).

De acordo com o Sistema Único de Saúde (SUS) a rede de atenção à saúde é feita a partir da adaptação de ações e serviços que buscam garantir a integralidade do cuidado, que tem como objetivo promover a integração dos serviços de saúde com incentivo à atenção continua, integral, de qualidade, responsável e humanizada, além de contribuir para a sedimentação dos princípios doutrinários do SUS (Lopes et al, 2017; Brasil, 2014; Brasil, 2018).

Nesse sentindo, a Atenção Primária em Saúde (APS) deve ser a porta de entrada da população, o que inclui os idosos e seus familiares ao serviço de saúde pública, e a partir da avaliação realizada nesse nível, associada à identificação das síndromes geriátricas presentes em cada indivíduo, deve ser definida a necessidade de referência dos mesmos para os outros níveis de cuidado (Santos et al, 2018). A estratificação da população idosa é definida a partir de perfis de funcionalidade, que planejam e organizam os objetivos do cuidado de cada pessoa acompanhada na rede de atenção à saúde (Almeida et al, 2020).

O perfil 1 é atribuído aos idosos independentes e autônomos para realizar as atividades de vida diária, sem necessidade de auxiliares ou auxiliadores, assim o atendimento desse indivíduo deve ser voltado essencialmente para a promoção e proteção da saúde. No entanto, essa classificação não exclui a possibilidade da necessidade de atendimento especializado, assim deve ser utilizado o serviço de referência e contrarreferência de acordo com a demanda relatada por cada idoso (Almeida et al, 2020; Ferreira et al, 2020). Já o perfil 2 enquadra o idoso que necessita de algum tipo de adaptação ou supervisão de um terceiro para realizar suas atividades de vida diária, o que faz com que sejam considerados pessoas em risco de se tornarem dependentes em algum momento, por isso o foco do cuidado deve ser a manutenção das funções executivas que ainda se encontram presentes, além de reabilitar as que estão com algum grau de comprometimento (Almeida et al, 2020; Ferreira et al, 2020).

O perfil 3 é constituído por idosos totalmente dependentes para realização das suas atividades diárias, o que determina a necessidade de acompanhamento contínuo, visando a prevenção terciária e quaternária, além do gerenciamento de comorbidades pré-existentes. Uma opção para esse tipo de demanda é o acompanhamento domiciliar planejado (Almeida et al, 2020; Ferreira et al, 2020).

Dessa forma, devido à alta prevalência de idosos na população brasileira, cerca de 9,82\% da população total segundo censo IBGE (2020), e o número diminuído de especialista em geriatria e gerontologia segundo o cadastro do Conselho Federal de Medicina (2019), faz-se necessária a implementação de estratégias que visem amenizar a demanda do idoso em consultórios especializados (Ferreira et al, 2020; Coelho, Motta \& Caldas, 2018).

Devido ao exposto, a sociedade e o sistema de assistência à saúde vivem um período de adaptação que exige mudanças em suas estratégias de atendimento. Mediante a isso, a fim de evitar a sobrecarga do sistema único de saúde, a organização do fluxo do paciente idoso deve ser padronizada de maneira a contemplar pacientes diagnosticados com as síndromes geriátricas e suas necessidades de encaminhamento para profissionais especialistas.

Considerando-se o cenário atual, esta pesquisa teve como objetivo principal criar um fluxograma para encaminhamento do paciente idoso com as síndromes geriátricas para uso do médico não especialista, além de validar através de julgamento dos geriatras atuantes na Clínica de Especialidades Médicas do Centro Universitário do Estado do Pará (CEMEC) após avaliar o grau de conhecimento técnico sobre a abordagem do paciente idoso dos profissionais médicos que trabalham no CEMEC. Posto isso, propõe-se a implementação de um fluxograma para melhor estruturação e orientação de profissionais não especialistas frente ao serviço de referência e contrarreferência, aprimorando o conhecimento desse profissional e a melhoria na qualidade e efetividade do atendimento do paciente idoso, a fim de garantir o seu envelhecimento saudável. 


\section{Metodologia}

Esta pesquisa foi aprovada pelo Comitê de Ética em Pesquisa em Seres Humanos (CEP) do Centro Universitário do Estado do Pará sob parecer consubstanciado número 4.500.172, emitido no dia 15 de janeiro de 2021.

Tratou-se de um estudo do tipo metodológico, de natureza aplicada, que incluiu pesquisa bibliográfica e abordagem quali e quantitativa, realizado no ambulatório de saúde do idoso do Centro de Especialidades Médicas do Centro Universitário do Estado do Pará (CEMEC), na cidade de Belém - PA com população alvo, composta por 44 médicos não-geriatras vinculados à instituição durante o período de execução da pesquisa e três médicos geriatras também vinculados e em desenvolvimento de atividades.

Esses profissionais médicos escolhidos não foram pesquisados a respeito de informações sociodemográficas como: sexo, idade, religião, tempo de atuação nas suas respectivas especialidades. Tais dados não foram necessários em virtude de que o estudo não teve como objetivo avaliar esses assuntos na população pesquisada, e sim o grau de conhecimento, já que se trata de um grupo de médicos professores e preceptores em Instituição de Ensino Superior, que estão em plena atividade de suas funções e completamente inseridos neste cenário.

O estudo constituiu-se por três etapas. A primeira etapa foi um estudo transversal analítico, através de um questionário autoaplicável para o médico não-geriatra, que avaliou o grau de conhecimento do profissional quanto ao encaminhamento correto do paciente idoso a um ambulatório especializado, por meio de cinco questões objetivas, capazes de analisar os seguintes tópicos: síndromes geriátricas, testes de triagem do paciente idoso e os critérios de encaminhamento.

A segunda etapa foi a criação de um fluxograma para orientar os profissionais não-geriatras ao encaminhamento correto do paciente idoso ao ambulatório de saúde do idoso tendo como base de formulação o Protocolo de Atenção à Saúde do Idoso (Brasil, 2014), modelos pré-existentes de definição das síndromes geriátricas, como os propostos pela Secretaria de Saúde do Estado do Paraná (2018) e pelos autores Moraes, Marino \& Santos (2010) e Lopes et al (2017) e os resultados obtidos dos questionários respondidos pelos médicos não especialistas.

Por fim, a terceira etapa, realizou-se mediante aplicação de questionário aos médicos especialistas a fim de validar o fluxograma criado na segunda etapa, avaliando o seu objetivo, conteúdo, linguagem, estrutura e aplicabilidade, para que após sua implementação, melhorou o fluxo de atendimento estabelecido pelo serviço de referência e contrarreferência.

Considerando um erro máximo de conclusão igual a 10\% (bilateral), um Intervalo de Confiança (IC) de 90\% e fazendo a estimativa total de médicos não especialistas atuantes no CESUPA, no período de realização da coleta de dados, de 155, a amostra do projeto em relação ao médico não especialista foi de 49. Além disso, para validação a amostra foi selecionada de maneira intencional, sendo composta por três médicos especialistas, número amostral justificado pela utilização da técnica Delphi e pelo total de médico geriatrias vinculadas ao local da pesquisa.

Foi avaliado o grau de conhecimento técnico-científico do médico generalista através de um questionário via google forms, visando auxiliar a criação do fluxograma. Além disso, a validação do fluxograma criado foi obtida pelos questionários também respondidos via google forms, por médicos especialistas em geriatria, atuantes no CEMEC.

Foram incluídos na aplicação dos questionários os médicos especialistas e não-geriatras regularmente vinculados ao Centro Universitário do Estado do Pará, e que estiverem desenvolvendo atividades no CEMEC no período de realização da pesquisa e aceitarem participar desta, por meio da assinatura do Termo de Consentimento Livre e Esclarecido, disponibilizado via online.

$\mathrm{Na}$ primeira etapa, foram excluídos os médicos não-geriatras vinculados ao CEMEC, que no período da coleta de dados estiverem afastados de suas funções com a instituição. Na terceira etapa, foi excluído o geriatra vinculado ao CESUPA que esteve orientando o trabalho para que não gere conflito de interesse com a pesquisa, e especialista com menos de seis 
meses de experiência clínica.

No questionário para o médico não-geriatra foram avaliados por meio de cinco questões objetivas o conhecimento técnico deste profissional, tendo como foco a avaliação de conhecimento dos testes de triagem do paciente idoso, das síndromes geriátricas e dos critérios de encaminhamento, analisando também a especialidade do profissional e se ele já encaminhou um paciente idoso para o ambulatório de saúde do idoso.

No questionário autoaplicável para os médicos geriatras foram questionadas opiniões a respeito do objetivo, conteúdo, linguagem e aplicabilidade do fluxograma proposto, que será analisada através da abreviação que melhor corresponda a opinião do profissional avaliador, sendo interpretada como inadequada, parcialmente adequada, adequada e totalmente adequada, em seguida será ofertado um espaço para sugestões quanto a estrutura e conteúdo do fluxograma apresentado.

As informações da caracterização amostral foram apuradas e digitadas em planilha elaborada no software Windows Microsoft Office Excel® 2016. Na aplicação da estatística descritiva foram construídos tabelas e gráficos para apresentação dos resultados em frequências e proporções. A estatística analítica foi utilizada para avaliar os resultados das respostas do questionário através dos Testes G e Qui-Quadrado Aderência para tabelas simples e Independência para comparação entre acertos e erros das questões.

Para validação do fluxograma foi calculado o IVC - Índice de Validação de Conteúdo e o Alfa de Cronback para avaliação da consistência do questionário aplicado. As estatísticas descritiva e analítica foram realizadas no software BioEstat ${ }^{\circledR} 5.4$ (versão gratuita, compatível com Windows). Para a tomada de decisão, foi adotado o nível de significância $\alpha=$ 0,05 , sinalizando com asterisco $(*)$ os valores significantes.

\section{Resultados}

Primeira Etapa: Análise do questionário dos médicos não-geriatras

Foram analisados 44 questionários que foram respondidos por médicos vinculados ao CESUPA, no entanto o link para o formulário foi encaminhado para um total 78 profissionais, sendo que três deles não quiseram responder (justificaram que a prática clínica em determinadas especialidades diminui o conhecimento geral quanto aos outros assuntos, tornando-os não qualificados para responder as questões disponibilizadas), um teve problemas quanto à abertura do link (o qual foi proposto auxílio ao acesso, porém o profissional alegou falta de tempo), e 30 não responderam a solicitação.

Dessa forma, primeiramente foi coletada a especialidade atuante pelos profissionais que aceitaram responder o formulário, assim, foi obtido respostas de 20 especialidades diferentes (Tabela 1). 
Tabela 1 - Relação das especialidades médicas que participaram da pesquisa.

\begin{tabular}{lcc}
\hline \multicolumn{1}{c}{ Qual a sua especialidade? } & & \\
\hline \hline Pediatria & 11 & $25,0 \%$ \\
Clínica Médica & 4 & $9,1 \%$ \\
Generalista & 4 & $9,1 \%$ \\
Medicina de Família e Comunidade & 4 & $9,1 \%$ \\
Cirurgia Vascular & 2 & $4,5 \%$ \\
Ginecologia e Obstetrícia & 2 & $4,5 \%$ \\
Pneumologista & 2 & $4,5 \%$ \\
Reumatologia & 2 & $4,5 \%$ \\
Endocrinologia & 2 & $4,5 \%$ \\
Anestesiologia & 1 & $2,3 \%$ \\
Cardiologia pediátrica & 1 & $2,3 \%$ \\
Cirurgia Digestiva & 1 & $2,3 \%$ \\
Cirurgia Oncológica & 1 & $2,3 \%$ \\
Endoscopia e gastroenterologia & 1 & $2,3 \%$ \\
Medicina intensiva & 1 & $2,3 \%$ \\
Nefrologia pediátrica & 1 & $2,3 \%$ \\
Neurologia & 1 & $2,3 \%$ \\
Oncologia pediátrica & 1 & $2,3 \%$ \\
Otorrinolaringologista & 1 & $2,3 \%$ \\
Reumatologia pediatra & 1 & $2,3 \%$ \\
Total & 44 & $100 \%$ \\
\hline & & \\
\hline
\end{tabular}

Fonte: Autores (2021).

Na Figura 1, observa-se que, do total dos 44 médicos não especialistas que responderam ao questionário, apenas dois deles $(4,5 \%)$ acertaram todas as questões, direcionadas ao conhecimento sobre as síndromes geriátricas, teste de triagem e critérios de encaminhamento do paciente idoso, sendo que este profissional afirmou também já ter tido encaminhado algum paciente para o ambulatório de geriatria. A maior proporção de acertos (43,2\%) foi observada para 4 das 5 questões respondidas ( $\mathrm{n}=19$ profissionais), aferindo proporção significante na quantidade de participante que tiveram 4 acertos. 
Figura 1 - Proporção do total de acertos nas questões sobre o grau de conhecimento técnico dos médicos não especialistas em geriatria vinculados ao CEMEC.

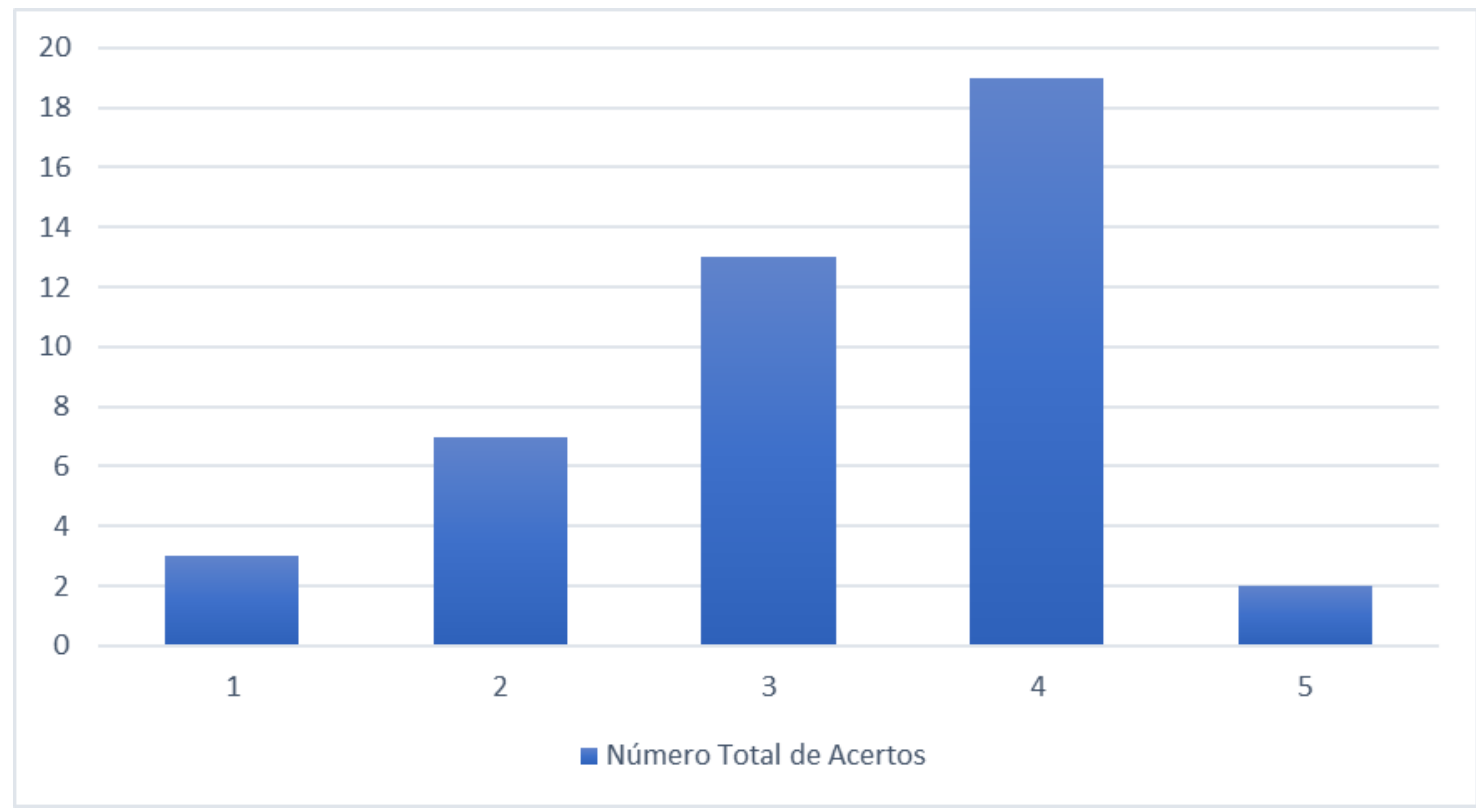

Fonte: Autores (2021).

A primeira questão tinha como tema principal a síndrome geriátrica de Incapacidade cognitiva e seus testes de triagem, com 40 respostas corretas, demonstrando que essa questão foi a que apresentou maior índice de acerto (90,9\%) (Tabela 2).

Tabela 2 - Índice de Acerto da Primeira Questão.

Seu José de 70 anos, com ensino médico completo, é levado a consulta pelo seu neto, que refere que
há cerca de 2 anos, o avô apresenta alguns lapsos de memória, e que isso vem piorando. Refere ter
começado a apresentar dificuldade em fazer as compras no supermercado e esquece onde coloca
alguns objetos pessoais, o que nunca tinha acontecido. É totalmente independente, mas ultimamente
em conversas com amigos no meio de uma frase, esquece algumas palavras, e acaba se irritando.
Nega alteração do sono e apetite. E apresenta como comorbidade hipertensão arterial em uso
contínuo de losartana. De acordo com o quadro, qual possível síndrome geriátrica o seu José se
encaixaria e quais testes poderia contribuir para o seu diagnóstico:

Síndrome da Incapacidade Cognitiva, mini exame do estado mental e teste do relógio. * $40 \quad 90,9 \%$

Síndrome da incontinência, mini exame do estado mental e time get up and go. $\quad 2 \quad 4,5 \%$

Síndrome de variação do humor, time get up and go e escala geriátrica da depressão. $\quad 1 \quad 2,3 \%$

Síndrome da Imobilidade, escala de Lawton e escala de Katz. $\quad 1 \quad 2,3 \%$

\begin{tabular}{lll} 
Síndrome da Instabilidade Postural, escala de Katz e teste do relógio. & 0 & $0,0 \%$ \\
\hline
\end{tabular}

$* \mathrm{p}<0.0001$ Teste G Aderência

Fonte: Autores (2021).

A segunda questão visava o reconhecimento do teste de triagem mais indicado entre as alternativas, para sugerir o diagnóstico de depressão. Esta apresentou índice de acerto de 79,5\%. (Tabela 3) 
Tabela 3 - índice de Acerto da Segunda Questão.

Dona Dalva, 70 anos, acompanhada da filha, relata que a cerca de 10 semanas percebeu a mãe está apática, ficou sem tomar banhos por 4 dias, não está se alimentando, dorme na maior parte do seu dia e estar sem fazer as atividades diárias por não se sentir disposta para tal. Não responde com clareza as perguntas feitas durante a anamnese e fala baixo. De acordo com esse quadro, qual teste poderia ser utilizado para esclarecer o diagnóstico:

\begin{tabular}{ccc}
\hline \hline Escala geriátrica da depressão* & 35 & $79,5 \%$ \\
Escala de Ramsay & 4 & $9,1 \%$ \\
Teste de fluência verbal & 4 & $9,1 \%$ \\
Time get up and go & 1 & $2,3 \%$ \\
Escala MRC modificada & 0 & $0,0 \%$ \\
\hline
\end{tabular}

*p $<0.0001$ Teste G Aderência

Fonte: Autores (2021).

A terceira questão apresentou o maior índice de erro $(90,9 \%)$, entre as 5 questões. Esta questão apresentava informações sobre características da síndrome geriátrica de instabilidade postural, apresentando como resposta correta que "a quantidade de medicamentos não influencia diretamente no risco de quedas, apenas a classe do medicamento influência nas quedas", porém a alternativa mais marcada foi a qual apresentava o teste de Romberg como útil apenas para avaliação de distúrbios da marcha, sendo escolhida por 20 médicos não geriatras. (Tabela 4)

Tabela 4 - Índice de Acerto da Terceira Questão.

\section{Em relação a instabilidade postural, qual a alternativa correta?}

\begin{tabular}{lcc}
\hline \hline $\begin{array}{l}\text { O teste de Romberg é útil apenas para a identificação de pacientes com } \\
\text { distúrbio de marcha* }\end{array}$ & 20 & $45,5 \%$ \\
$\begin{array}{l}\text { O teste Timed up and go de } 9 \text { segundos indica que o paciente tem uma } \\
\text { mobilidade restrita. }\end{array}$ & 10 & $22,7 \%$ \\
$\begin{array}{l}\text { Pacientes do sexo masculino caem com mais frequência do que pacientes do } \\
\text { sexo feminino. }\end{array}$ & 6 & $13,6 \%$ \\
Os anti-inflamatórios não hormonais estão associados ao risco de queda. & 4 & $9,1 \%$ \\
A quantidade de medicamentos não influencia diretamente no risco de \\
quedas, apenas a classe do medicamento influência nas quedas.
\end{tabular}

$* p=0.0011$ Teste G Aderência

Fonte: Autores (2021).

A quarta questão abordava sobre os critérios que justificariam o encaminhamento de um paciente idoso ao geriatra, levando em consideração sua idade, suas comorbidades e medicamentos em uso. Dessa forma, esse encaminhamento se justificaria pela seguinte assertiva: "Idade > 60 anos, com incontinência urinária acompanhada de prejuízo social”, que foi escolhida por $68,2 \%$ dos indivíduos, pois além de apresentar idade suficiente para ser acompanhada na geriatria, a paciente ainda apresentava uma síndrome geriátrica que lhe acarretava prejuízos na sua vida social, necessitando assim de um acompanhamento especializado. (Tabela 5) 
Tabela 5 - Índice de Acerto da Quarta Questão.

Idosa C.P.L de 68 anos, sexo feminino, hipertensa e dislipidêmica, com níveis de colesteróis e pressão arterial bem controlada, em uso de losartana e rosuvastatina, apresentando fratura em punho decorrente de queda de uma escada de 5 degraus, além disso, relata que ao tossir, espirrar e se levantar pela manhã perdia pequena quantidade urinária, isto a atrapalha no convívio com amigos e família. Quais das seguintes opções justifica o encaminhamento da paciente ao geriatra:

Idade > 60 anos com incontinência urinária acompanhado de prejuízo social.*

$30 \quad 68,2 \%$

Idade > 60 anos associado a hipertensão e dislipidemia

$12 \quad 27,3 \%$

Hipertensão e Dislipidemias

$1 \quad 2,3 \%$

Fratura em punho

O uso de dois medicamentos

$0 \quad 0,0 \%$

$* \mathrm{p}<0.0001$ Teste G Aderência

Fonte: Autores (2021).

A quinta questão apresentava um quadro de Doença de Alzheimer, e o índice de acerto desta questão foi de $75 \%$, porém a segunda assertiva mais marcada $(20,4 \%)$ foi que qualquer preocupação com idosos, justificaria esse encaminhamento (Tabela 6).

Tabela 6 - índice de Acerto da Quinta Questão.

Mulher de 76 anos, há 2 anos vem apresentando esquecimento para fatos rotineiros, chegou a esquecer até uma panela no fogo. Há uma semana, saiu sozinha para ir ao supermercado e acabou se perdendo. Relatou ainda não apresenta comorbidades. Após isso, família resolveu marcar uma consulta com o geriatra. Dessa forma, o atendimento geriátrico era corretamente indicado nessa situação?

Sim, pois a paciente provavelmente apresenta um quadro de doença de Alzheimer* $3375,0 \%$

Sim, pois qualquer preocupação em idosos, eles devem ser consultados por um geriatra. $920,4 \%$

Não, pois o quadro apresentado ainda é leve.

Não, o geriatra não é um médico recomendado neste caso.

$12,3 \%$

$* \mathrm{p}<0.0001$ Teste G Aderência

Fonte: Autores (2021).

Segunda Etapa: Criação do fluxograma para encaminhamento do paciente idoso para uso do médico não geriatra 
Figura 2 - Fluxograma de Encaminhamento do Paciente Idoso Para Uso do Médico Não-geriatra.

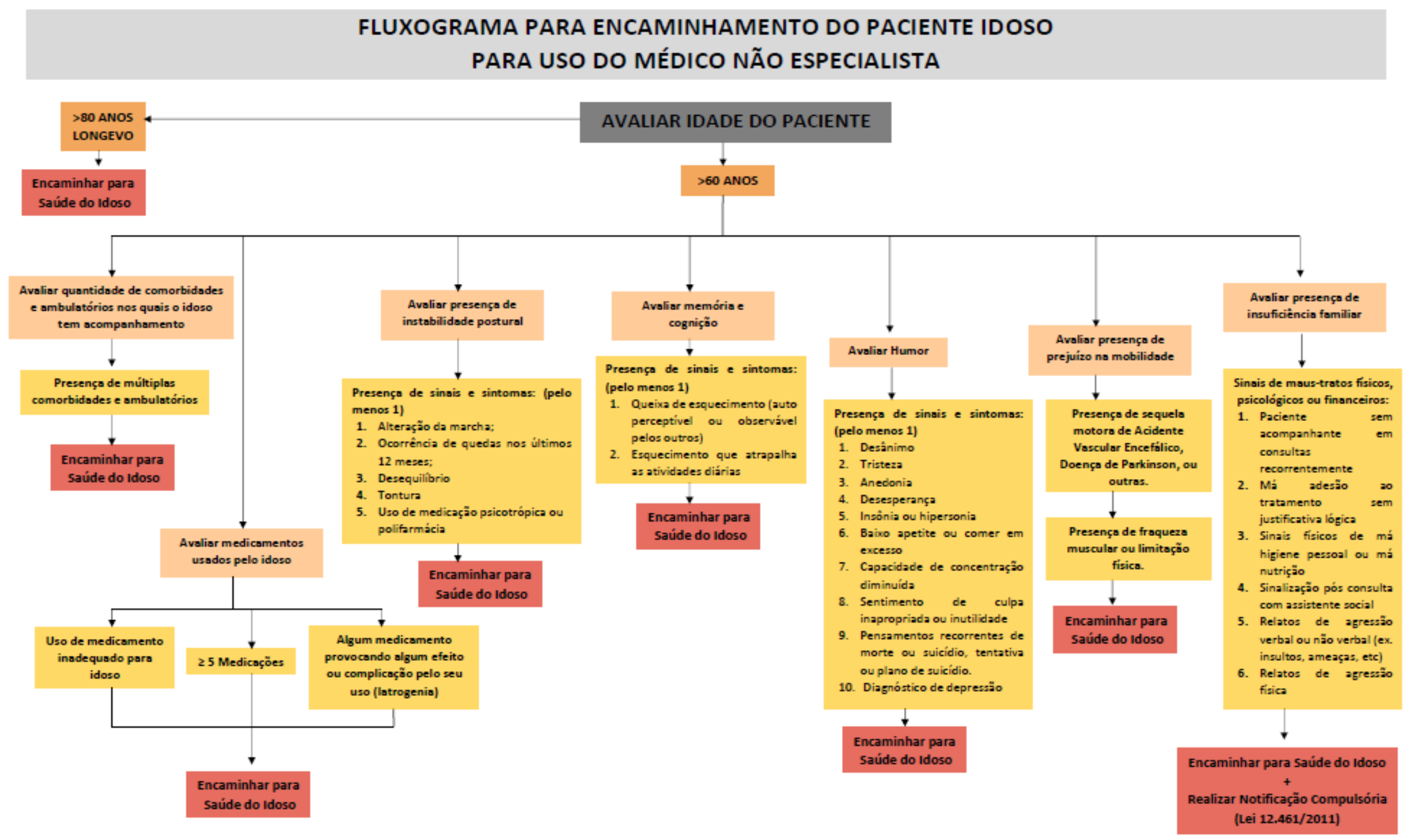

Fonte: Autores (2021). 


\section{Terceira Etapa: Validação do Fluxograma.}

Por meio de um questionário tipo Likert, disponibilizada via google forms, através da Técnica de Delphi que leva em consideração a opinião do especialista, respondido por 3 médicos geriatras atuantes no CESUPA, foi valido o fluxograma criado quanto ao seu conteúdo, aplicabilidade, objetivos e linguagem e estrutura, com porcentagem geral da avaliação de $84,4 \%$, alcançado o mínimo de $76 \%$ para que o mesmo seja validado. (Tabela 7).

Tabela 7 - Percentual de Validação do Fluxograma de Encaminhamento.

\begin{tabular}{ccccc}
\hline Domínios para validação & $\begin{array}{c}\text { Pontuação } \\
\text { Recebida }\end{array}$ & $\begin{array}{c}\text { \% } \\
\text { Aprovação }\end{array}$ & IVC & Cronbach \\
\hline \hline Conteúdo & 28 & $87,5 \%$ & 0,88 & ---- \\
Aplicabilidade & 14 & $87,5 \%$ & 1,00 & ---- \\
Objetivos & 26 & $81,3 \%$ & 1,00 & --- \\
Linguagem e Estrutura & 13 & $81,3 \%$ & 1,00 & ---- \\
Geral & $\mathbf{8 1}$ & $\mathbf{8 4 , 4 \%}$ & $\mathbf{0 , 9 6}$ & $\mathbf{0 , 8 9 8}$ \\
\hline
\end{tabular}

Fonte: Autores (2021).

\section{Discussão}

Nessa primeira etapa, analisando os questionários, pode-se aferir resultados significativos acerca dos encaminhamentos e conhecimentos dos médicos que referenciam os pacientes geriátricos ao ambulatório especializado. Inicialmente, foi possível verificar certa relutância dos profissionais de outras especialidades, quanto a responder o questionário, devido à falta de segurança em responder questões sobre assuntos não comuns em sua prática de trabalho.

Foi verificado que uma grande variedade de especialidades já encaminhou em algum momento da sua formação um paciente idoso ao ambulatório de geriatria. Com a análise dessas especialidades pode-se constatar que entre os 20, os que mais referiram encaminhar seus pacientes para geriatria foram de Clínica Médica e Medicina de Família e Comunidade. Esses médicos não especialistas em Geriatria atendem uma grande quantidade de pacientes, com diferentes idades, diagnósticos e realidades sociais, e que com aumento da expectativa de vida brasileira, tendem a atender cada vez mais a população idosa, tornando-se necessária maior orientação quanto ao possível seguimento, avaliação e necessidade de encaminhamento deste paciente (Côrte et al, 2017).

Esses médicos especialistas em Clínica médica e Medicina de Família e Comunidade foram as especialidades com maior aproveitamento técnico em relação as questões disponibilizadas tendo uma média aritmética de acerto de 4 questões, sendo igual a moda de aproveitamento geral de 4 questões.

Observou-se também que o maior índice de erro em questões foi a respeito da avaliação da instabilidade postural, na qual houve apenas 9,09\% de acerto, tendo ainda grande variabilidade nas alternativas escolhidas por cada profissional, não demonstrando um consenso quanto ao conhecimento técnico das características dessa síndrome. Essa informação é corroborada pelo trabalho de Carqueja et al (1999), no qual foi demonstrado que apesar dos sintomas de instabilidade postural serem os mais presentes em paciente em uma enfermaria de Clínica Médica em um hospital universitário em Florianópolis SC, não foram relatados nenhuma vez em fichas médicas, apenas em registros da enfermagem, demonstrando a presença de um 
problema.

Outro ponto importante, foi que a questão que abordava os testes de triagem e síndrome da incapacidade cognitiva foi a que teve o maior percentual de acerto $(90,7 \%)$. Essa questão contava com informações epidemiológicas e presença de sinais e sintomas que orientavam o raciocínio clínico, para o possível diagnóstico e conduta do caso.

Ademais, na última questão que discorria sobre os critérios de encaminhamento do idoso e doença de Alzheimer, a segunda alternativa mais escolhida foi a que afirmava que "em qualquer preocupação em idosos, eles devem ser consultados por um geriatra", o que demonstra certa tendência a sobrecarga dos ambulatórios especializados em geriatria, já que nem todo paciente tem real necessidade desse atendimento mais especializado, podendo sem acompanhado em unidades de saúde, por exemplo, com médicos de família e comunidade (Oliveira, Veras \& Cordeiro, 2019).

Na segunda etapa, o fluxograma de encaminhamentos interespecialidades, que foi criado a partir dos resultados desta pesquisa, teve seu conteúdo e estrutura gráfica fundamentado com informações e outros modelos que expunham testes de triagem, sinais e sintomas e protocolos de encaminhamento específicos (Moraes \& Santos, 2010; Apóstolo, 2012; Secretaria de Saúde do Estado do Espírito Santo, 2016)

O principal benefício desse fluxograma, é a facilidade e rapidez em obter um direcionamento geral para avaliação e seguimento do paciente, em relação as principais queixas e sinais presentes em um atendimento com o paciente idoso (Moraes \& Santos, 2010; Apóstolo, 2012; Ferreira et al, 2017).

Nesse sentindo, apoiado pelos resultados encontrados, este fluxograma permite uma tomada de decisão clínica mais acurada, rápida e eficiente para as queixas geriátricas, minimizando a sobrecarga do ambulatório especializado, além de minimizar erros de encaminhamentos. Além de ser um guia para médicos que não atendem rotineiramente os pacientes idosos, porém que devido sua especialidade ficam vulneráveis a avaliação desse paciente e possível necessidade de encaminhamento dele.

$\mathrm{Na}$ terceira etapa, para validação do fluxograma, a partir da coleta das opiniões e avaliações dos médicos geriatras atuantes no CEMEC, foi percebido a existência de alguns critérios de encaminhamento pré-existente no ambulatório de saúde do idoso, porém que não eram de conhecimentos dos outros ambulatórios, o que desfavorecia a ocorrência do encaminhamento adequado do idoso ao ambulatório especializado.

Além disso, após a análise da avaliação de cada item do fluxograma foi percebido que os critérios colocados em evidência para encaminhamento do paciente idoso eram pertinentes e tornavam mais eficientes a identificação dos sintomas ou sinais que sinalizam a necessidade do encaminhamento do paciente geriátrico. Nesse sentido, houve concordância quanto a validação do fluxograma criado pelos três juízes, atingindo mais de $76 \%$ de aprovação globalmente, nos 5 critérios avaliados: objetivo, conteúdo, aplicabilidade, linguagem e estrutura. Além de ter aprovação em cada critério isoladamente. Reafirmando os benefícios que podem ser oferecidos se o fluxograma for usado rotineiramente no CEMEC, como encaminhamentos mais corretos e diminuição da sobrecarga de atendimento no ambulatório de saúde do idoso.

Tais achados podem ser generalizados por outros serviços, pois utilizam termos e conhecimentos difundidos por consensos e diretrizes, além de tornar prático e dinâmico este fluxo de encaminhamentos. Recomenda-se a aplicabilidade do fluxograma para o benefício de evitar sobrecarga dos serviços em saúde de geriatria.

\section{Conclusão}

Infere-se, portanto, que dos 44 médicos não geriatras que participaram do estudo, a maioria, isto é 29, já encaminharam em algum momento da sua vida profissional um paciente idoso a um ambulatório de geriatria. Sendo que esses 
Research, Society and Development, v. 10, n. 12, e129101220304, 2021

(CC BY 4.0) | ISSN 2525-3409 | DOI: http://dx.doi.org/10.33448/rsd-v10i12.20304

44 profissionais eram de 20 especialidades médicas diferentes, reafirmando a associação de patologias geriátricas com diversas especialidades clínicas e cirúrgicas. Ademais, a investigação do conhecimento técnico dos profissionais, demonstrou maior conhecimento quanto a síndrome geriátrica de incapacidade cognitiva e seus testes de triagem.

No entanto, houve um alto percentual de erro quanto a síndrome de instabilidade postural, demonstrando a utilidade da melhor orientação quanto a necessidade de avaliação especializada em um paciente idoso que apresentam sinais ou sintomas que sinalizem a presença dessa síndrome.

Diante do exposto e dos dados obtidos, observa-se a necessidade da implementação do fluxograma para encaminhamento do paciente idoso para médicos não especialistas devido à ausência de um guia que melhore a qualidade dos encaminhamentos, a fim de padronizá-los e diminuir o índice de encaminhamentos incorretos, a fim de reduzir a demanda do ambulatório de saúde do idoso e aumentar o número de vagas para atendimento de idosos que realmente necessitam do atendimento especializado.

O produto validado torna-se instrumento não somente para uso na prática em cenários reais como também para novas pesquisas e estudos para aprimorar, ajustar ou avaliar aplicabilidade desse fluxograma no ambiente ambulatorial.

\section{Referências}

Agência de Notícias IBGE. (2019). Em 2018, expectativa de vida era de 76,3 anos. https://agenciadenoticias.ibge.gov.br/agencia-sala-de-imprensa/2013agencia-de-noticias/releases/26104-em-2018-expectativa-de-vida-era-de-76-3-anos >.

Aires, I. O. et al. (2019). Food consumption, lifestyle and its influence on the aging process. Research, Society and Development, 8 (11), e098111437. DOI: 10.33448/rsd-v8i11.1437. https://rsdjournal.org/index.php/rsd/article/view/1437.

Almeida, A. P. S. C. et al. (2020). Falta de acesso e trajetória de utilização de serviços de saúde por idosos brasileiros. Ciências e saúde coletiva, 25 (6), https://doi.org/10.1590/1413-81232020256.27792018

Apóstolo, J. L. A. (2012). Instrumentos para avaliação em geriatria (Geriatric Instruments). Escola Superior de Enfermagem de Coimbra. Coimbra.

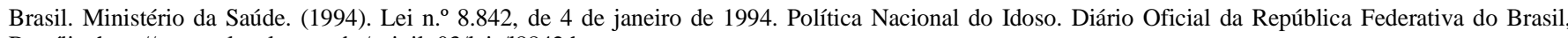
Brasília. http://www.planalto.gov.br/ccivil_03/leis/18842.htm

Brasil. Ministério da Saúde. (2013). Estatuto do Idoso. Brasília. https://bvsms.saude.gov.br/bvs/publicacoes/estatuto_idoso_3edicao.pdf

Brasil. Ministério da Saúde. (2014). Protocolo de Atenção à Saúde do Idoso. Governo do Distrito Federal. https://www.saude.df.gov.br/wpconteudo/uploads/2018/04/Protocolo_Saude_do_Idoso.pdf

Brasil. Ministério da Saúde. (2014). Diretrizes para o cuidado das pessoas idosas no SUS: proposta de modelo de atenção integral. https://bvsms.saude.gov.br/bvs/publicacoes/diretrizes_cuidado_pessoa_idosa_sus.pdf

Carqueja, C. L. et al. (1999). Detecção de problemas clínico-sociais de pacientes geriátricos internados nas enfermarias de clínica médica do Hospital Universitário. Trabalho de Conclusão de Curso. https://repositorio.ufsc.br/bitstream/handle/123456789/118355/226737.pdf?sequence=1

Coelho, L. P., Motta, L. B., \& Caldas, C. P. (2018). Rede de atenção ao idoso: fatores facilitadores e barreiras para implementação. PHYSIS/Rio de Janeiro, 28, e280404. https://doi.org/10.1590/S0103-73312018280404

Côrte, B., Kimura, C., Ximenes, M. A.; \& Nóbrega, O. T. (2017). Determinantes da atenção aos idosos pela rede pública de saúde, hoje e em 2030: o caso da Região Metropolitana de São Paulo. Saud soc. 26 (3). https://doi.org/10.1590/S0104-12902017159606

Damasceno, M. J. C. F.; \& Chirelli, M. Q. (2019). Implementação da Saúde do Idoso na Estratégia Saúde da Família: visão dos profissionais e gestores. Ciênc. saúde colet. 24 (5), 1637-1646. https://doi.org/10.1590/1413-81232018245.04342019

Ferreira, L. S. et al. (2020). Acesso à Atenção Primária à Saúde por idosos residentes em zona rural no Sul do Brasil. Rev Saúde Pública, 54. https://doi.org/10.11606/s1518-8787.2020054002316

Ferreira, L. V. et al. (2017). Busca do autocuidado por idosos na rede de atenção à saúde. Revista Contexto \& Saúde, 17 (32), $46-54$.

Freitas, E. V. de; \& Py, L. (2017). Tratado de Geriatria e Gerontologia. $4^{\mathrm{a}}$ ed. Rio de Janeiro. Guanabara Koogan.

Lopes, P. de C. et al. (2017). Estilo de vida e intervenções não farmacológicas no tratamento e na prevenção das síndromes geriátricas: uma revisão integrativa. Revista Kairós: Gerontologia, 20 (2), 375-398.

Moraes, E. N. de; Marino, M. C.; Santos, R. R. (2010). Principais síndromes geriátricas. Minas Gerais, 20 (1), 54-56. 
Research, Society and Development, v. 10, n. 12, e129101220304, 2021

(CC BY 4.0) | ISSN 2525-3409 | DOI: http://dx.doi.org/10.33448/rsd-v10i12.20304

Oliveira, M. R., Veras, R. P., Cordeiro, H. de A. (2019). Physis 28 (04). https://doi.org/10.1590/S0103-73312018280411

Sandri, J. V. de A. et al. (2019). Políticas do Idoso e Atendimento Multidisciplinar na Área Da Saúde. Anais do Encontro Mãos de Vida, 5 (1).

Santos, M. S. et al. (2018). Práticas integrativas e complementares: avanços e desafios para a promoção da saúde de idosos. Revista Mineira de Enfermagem, 22:e-1125. DOI: $10.5935 / 1415-2762.20180048$

Secretaria de Saúde do Estado do Espírito Santo. (2016). Protocolos de Regulação para Acesso a Consultas e Exames Especializados. Vitória. https://saude.es.gov.br/Media/sesa/Protocolo/Protocolo\%20consultas\%20e\%20exames.pdf

Secretaria de Estado da Saúde do Paraná. (2018). Avaliação multidimensional do idoso. Curitiba. https://www.saude.pr.gov.br/sites/default/arquivos_restritos/files/documento/2020-04/avaliacaomultiddoidoso_2018_atualiz.pdf

World Health Organization. (2015). Relatório mundial sobre envelhecimento e saúde: resumo. Geneva http://apps.who.int/iris/bitstream/handle/10665/186468/WHO_FWC_ALC_15.01_por.pdf?sequence=6 